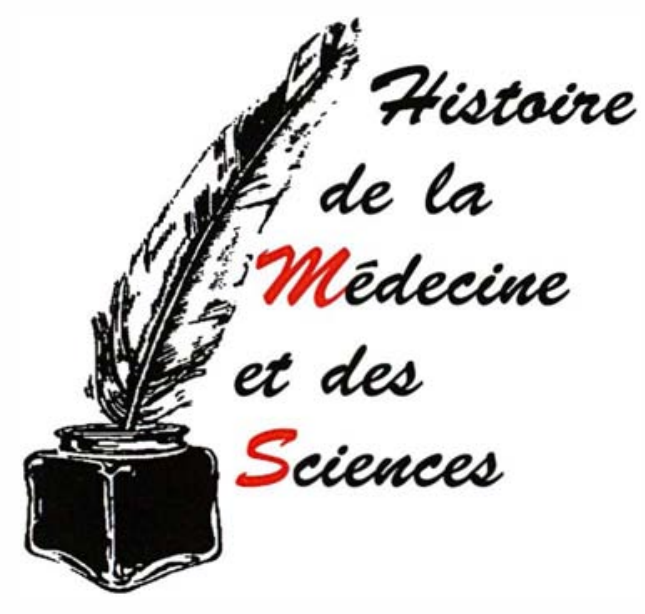

Est-ce bien connaître la Science que de n'en connaître que les aboutissements, et non le cheminement, les hésitations et les chemins de traverse qu'elle emprunte souvent? Des erreurs ne seraientelles pas évitées si les erreurs passées étaient présentes à l'esprit, des espoirs inconsidérés ne sembleraient-ils pas bien irréalistes à qui considérerait tous ceux qui se sont évanouis comme des illusions passagères dans le passé ? Peut-on aimer la Science sans aimer ceux qui la font, peut-on admirer les chercheurs d'antan sans savoir ce que furent les conditions, matérielles, intellectuelles et affectives de l'élaboration de leurs hypothèses et du déroulement de leur démarche? Autant de questions contenant leurs réponses, et autant de réponses qui nous ont fait désirer depuis longtemps proposer à nos lecteurs une nouvelle rubrique à dimensions historique et épistémalogique. Faisant cela, et sans goût aucun du paradoxe, nous avons le sentiment de rester fidèles à notre objectif initial qui est de nous placer sur le firont mowant de la comaissance, entre Médecine et Science. Comme le rappelle Michel Morange dans la première contribution à cette rubrique, c'est le sens qui donne aux informations le statut d'une connaissance, et ce sens ne peut en effet souvent être approché qu'à la lumière d'une perspective historique et épistémologique. Les contributions qui nous sont soumises pour alimenter cette rubrique seront évaluées sur le plan de leur qualité et de leur pertinence, tout en laissant naturellement aux auteurs une totale liberté d'analyse et de propos, dès lors que leur texte obéira aux critères exigibles de véracité et de cohérence, évitant toute tonalité anormalement polémique et agressive. A vos plumes...!

$\mathrm{m} / \mathrm{s} n^{\circ} 8$, vol. 11 , aoüt 95

\title{
De la découverte du rôle de I'ADN au drame du sang contaminé: ce que savoir veut dire
}

es ouvrages qui décrivent l'histoire de la biologie moléculaire mentionnent deux expériences ayant démontré que les gènes étaient formés d'ADN : la première, dont les résultats furent publiés en 1944 par Oswald Avery, Colin MacLeod et Maclyn McCarthy [1], montrait que le facteur transformant des pneumocoques, capable in vitro de modifier la capsule polysaccharidique qui entoure cette bactérie, était de l'ADN pur; la deuxième, réalisée par Alfred Hershey et Martha Chase en 1952, démontrait que lors de la multiplication d'un bactériophage, seul l'ADN du phage pénétrait dans la bactérie et était donc responsable de la reproduction de celui-ci [2].

L'existence de deux expériences, séparées par huit années, est expliquée par le peu d'impact que le résultat d'Avery aurait eu sur la communauté des biologistes. Publiée dans une revue médicale non lue par les généticiens, avec un titre peu attractif et une discussion très prudente, l'expérience d'Avery serait passée inaperçue. Ceux qui l'auraient connue, tels les collègues d'Avery à l'Institut Rockefeller, en auraient même mis en doute le caractère démonstratif.

A l'issue d'un lent et patient travail de recherche historique, le biologiste anglais H.V. Wyatt [3] a montré qu'Avery lui-même avait bien vu l'importance de sa découverte pour la génétique [4], que cette expérience n'avait pas été ignorée des principaux acteurs de la jeune biologie moléculaire, et que ses résultats avaient été présentés dans de nombreuses revues et articles de vulgarisation. S'il est vrai que son caractère démonstratif fut mis en cause, la comparaison avec la deuxième expérience, celle d'Hershey et Chase, est cependant édifiante: la démonstration d'Avery apparaît bien mieux fondée, bien plus "propre", que celle fournie par le groupe du phage [5]. L'explication proposée par Wyatt pour justifier le peu d'impact de l'expérience d'Avery est donc autre. Le résultat de ce dernier a bien constitué une information. Cette information n'a cependant pas pu être incorporée aux idées alors acceptées sur la nature du matériel héréditaire, la structure de l'ADN ou le fonctionnement des organismes bactériens. Cette information n'a pu être convertie en connaissance. Avery effectue en effet ses recherches dans un domaine (la bactériologie) qui est périphérique par rapport aux intérêts majeurs des généticiens. En outre, la révélation d'une fonction génétique de l'ADN est ininterprétable: d'une part, cette molécule a, selon les spécialistes, une structure chimique monotone; d'autre part, personne ne voit le lien "logique" qui pourrait exister entre la molécule d'ADN et le résultat de la transformation, la modification de la capsule polysaccharidique présente à la surface du pneumocoque [6]. Il faudra attendre que les travaux d'Erwin Chargaff - inspirés par les résultats d'Avery - montrent que la molécule 
d'ADN a une structure complexe et non monotone, et que la nouvelle vision informationnelle de la biologie moléculaire commence à se mettre en place, pour que le rôle génétique de l'ADN puisse être envisagé.

Cette démonstration de Wyatt révèle comment se construit la connaissance scientifique. Celle-ci résulte toujours d'un choix parmi des informations abondantes et contradictoires. A chaque époque, et encore aujourd'hui, les théories dominantes excluent un certain nombre d'observations, d'informations. Celles-ci sont acceptées en tant qu'informations, mais ne sont pas intégrées au corpus des connaissances: elles ne constituent pas un savoir. Elles ne peuvent conduire, ni à un programme de recherches [7], ni à un programme d'actions.

Le rappel de la distinction entre information et connaissance est particulièrement important à notre époque où l'on identifie souvent, à tort, la quantité toujours croissante d'informations à notre disposition avec un progrès des connaissances. Elle peut être aussi utile pour porter un nouveau regard sur un drame majeur de ces dernières années, l'affaire du "sang contaminé" et la recherche des responsabilités impliquées. Presque tous les récits de ces événements entretiennent une confusion totale entre les informations dont on pouvait disposer à un moment donné, et la connaissance de la maladie qui en découlait [8-9]. Je ne prendrai qu'un exemple, parmi de nombreux possibles, de cette confusion : la signification de la séropositivité chez les individus ne présentant aucun signe clinique de la maladie. Dès 1984, l'analyse du sang des individus atteints du SIDA révèle la présence d'anticorps dirigés contre le virus maintenant appelé VIH [10]. Cette observation est fondamentale car elle démontre l'implication du VIH dans le développement du SIDA. Cependant, les anticorps sont aussi retrouvés dans le sang de nombreux individus épargnés par la maladie, mais dont on sait qu'ils ont pu être contaminés par le virus. Il s'agit là d'une infor- tée. La signification de la présence d'anticorps chez des individus "sains " est cependant ambiguë. Le fait que la séropositivité soit le signe annonciateur du développement de la maladie ne fut pas immédiatement accepté. Pendant quelques mois, la présence d'anticorps fut considérée comme un signe favorable, la marque du combat de l'organisme contre le virus. Le suivi des individus séropositifs montra cependant que la présence d'anticorps n'empêchait pas le développement de la maladie; malgré cette observation, beaucoup pensaient encore que la conversion de la séropositivité en maladie ne concernait qu'une fraction minime des individus touchés [11]. La triste réalité mit plusieurs années à s'imposer. L'information - les individus séropositifs développent le SIDA n'a donc pas été aussitôt une connaissance: les conséquences de cette information n'ont pas été immédiatement et intégralement prises en compte, utilisées, avec toute l'énergie qui eût été nécessaire, pour éliminer, par exemple, tout risque lié à la transfusion sanguine.

Pourquoi? Parce que les connaissances antérieures que l'on avait sur les maladies infectieuses étaient en contradiction avec ces informations nouvelles sur le virus du SIDA. On ne connaissait pas de maladies infectieuses tuant si efficacement, apparemment si lentement, après une très longue phase silencieuse, alors même que l'organisme avait réagi contre l'agent pathogène. Cette radicale nouveauté du virus du SIDA “explique» le délai qui a été nécessaire pour que les différentes informations concernant ce virus deviennent des connaissances.

La conversion des informations en connaissance est un processus lent, difficile, exigeant la réorganisation d'un champ cognitif. Comprendre cela, c'est reconnaître que la connaissance scientifique est une aventure humaine, et non le simple dévoilement d'une réalité absolue, indépendante de l'être humain. C'est aussi accepter que l'erreur, l' "aveuglement» devant l'évidence, soient des caractéristiques "normales» de la démarche scientifique

\section{Michel Morange}

École normale supérieure, unité de génétique moléculaire, 46, rue d'Ulm, 75230 Paris Cedex 05, France.

\section{Remerciements}

Je remercie mes collègues du GRC qui, par leurs discussions, ont joué un rôle important dans la préparation de ce manuscrit.

\section{RÉFÉRENCES}

1. Avery OT, MacLeod CM, McCarty M. Studies on the chemical nature of the substance inducing transformation of Pneumococcal types. $J$ Exp Med 1944; 79: 137-58.

2. Hershey $A D$, Chase $M$. Independent functions of viral proteins and of nucleic acids in the growth of the bacteriophage. $J$ Gen Physiol 1952; 36: 39-56.

3. Wyatt HV. When does information become knowledge? Nature $1972 ; 235$ : 86-9.

4. Ce qui fut confirmé par son collaborateur: McCarty M. The Transforming principle. New York: WW Norton Company, 1985.

5. Wyatt HV. How history has blended. Nature 1974; 249: 803-5.

6. Löwy I. Variances in meaning in discovery accounts: the case of contemporary biology. HSPS 1990; 21 : 87-121.

7. Wyatt HV. Knowledge and prematurity: the journey from transformation to DNA. Persp Biol Med 1975; 18: 149-56.

8. Sauf Grmek MD. Histoire du SIDA. Paris: Payot, 1989.

9. Miller G. Le SIDA, la médecine et l'opinion. La Recherche 1995; 272: 92-4.

10. Sarngadharan MG, Popovic M, Bruch L, Schüpbach J, Gallo RC. Antibodies reactive with human T-lymphotropic retroviruses (HTLVIII) in the serum of patients with AIDS. Science 1984; 224: 506-8.

11. Budiansky S. AIDS tests alarm blood banks. Nalure 1985; 313 : 87.

\section{TIRÉS À PART}

M. Morange. 